Volumen: 23 Fecha de recibido: 24/9/2020 Fecha de publicación: Enero-Junio de 2021

Año: 2021 Fecha de aceptado: 25/11/2020 Correo: revista.societas@up.ac.pa

Numero: $1 \quad$ Número de Páginas: 72-92

URL:https://revistas.up.ac.pa/index.php/societas

\title{
Una Mirada Reflexiva Hacia Las Prácticas De Los Docentes En La Educación Emergente
}

\author{
Elizabeth Román-Machado1 \\ Universidad Metropolitana de Educación, Ciencia y Tecnología, Panamá , Panamá \\ roman.ely@gmail.com \\ Orcid: https://orcid.org/0000-0001-7863-9793
}

\section{Liris E. Ogia B. ${ }^{2}$}

Universidad Metropolitana de Educación, Ciencia y Tecnología, Panamá, Panamá

liris.ogia@gmail.com

Orcid: https://orcid.org/0000-0002-1762-8238

"En cuestiones de cultura y de saber, solo se pierde lo que se guarda; solo se gana lo que se da".

Antonio Machado

\section{Resumen}

Este artículo propone una mirada reflexiva hacia la educación de alta vulnerabilidad que, con la aparición del Covid-19 y las medidas de confinamiento, no ha hecho más que profundizarse en comunidades caracterizadas por la precariedad material y cultural en toda Latinoamérica, y por ende en Colombia, en donde funcionan grupos escolares como la "Institución Educativa República de Venezuela", en la que sus docentes, estudiantes y familias han realizado un enorme esfuerzo por adaptarse a una compleja dinámica que no fue planificada. Por ello, se plantea la necesidad de rescatar las prácticas de la educación de emergencia por dos vías, la primera, por medio de la sistematización de experiencias para convertir en capacidades institucionales: acciones, rutinas y estrategias implementadas de manera efectiva en esta pandemia; y, la segunda, por la identificación de desaciertos, para

${ }^{1}$ Doctora en Ciencias de la Educación. Docente e investigadora. Universidad Metropolitana de Educación, Ciencia y Tecnología "Umecit”: Panamá. roman.ely@gmail.com - Orcid: https://orcid.org/0000-0001-7863-9793

${ }^{2}$ Doctoranda de la Universidad Metropolitana de Educación, Ciencia y Tecnología "Umecit": Panamá. Magister en Tecnologías de la Información Aplicadas a la Educación. liris.ogia@gmail.com - Orcid: https://orcid.org/0000-0002-1762-8238 
emprender su transformación a través de la Investigación-Acción con el apoyo de todos sus grupos de interés.

Palabras clave: prácticas docentes, sistematización de experiencias, investigación-acción, educación emergente.

\begin{abstract}
This article proposes a reflective look at highly vulnerable education that, with the appearance of Covid-19 and the confinement measures, has only deepened in communities characterized by material and cultural precariousness throughout Latin America, and therefore in Colombia, where school groups such as the "Institución Educativa República de Venezuela" operate, in which its teachers, students and families have made an enormous effort to adapt to a complex dynamic that was not planned. For this reason, there is a need to rescue emergency education practices in two ways, the first, through the systematization of experiences to convert into institutional capacities: actions, routines and strategies implemented effectively in this pandemic; and, the second, for the identification of mistakes, to undertake their transformation through Research-Action with the support of all its stakeholders.
\end{abstract}

Keywords: teaching practices, systematization of experiences, Action-Research, emergency education.

\title{
Introducción
}

La irrupción del Covid-19 generó medidas de distanciamiento físico que obligaron en todo el mundo a la suspensión de las actividades escolares regulares, y su posterior reactivación con diversas estrategias extraordinarias: compactación de los programas académicos, guías de aprendizaje autodirigido o para ser orientado por padres o cuidadores, clases por radio y televisión, plataformas tecnológicas como Moodle, Microsoft Team, Google Classroom entre otras, o por medio del móvil con aplicaciones como WhatsApp. Estas medidas han demostrado que la brecha digital entre estratos socioeconómicos no solo está presente, sino que se profundiza y perjudica en mayor medida, como es obvio, a los sectores más desposeídos. Esto genera alta vulnerabilidad educativa, es decir, una situación en la cual muchos estudiantes por tiempo indeterminado no están recibiendo los beneficios de un currículo y un espacio áulico con condiciones al menos esenciales para un proceso formativo (Díaz y Pinto, 2017).

En Latinoamérica, esta situación ha impactado de forma muy notoria, ya que en las grandes ciudades es donde hay mayor riesgo de contagio y en las periferias suela haber una limitada disposición a conexión de Internet, con diferencias significativas entre los países de esta región, donde las medidas han sido: continuar con "normalidad" las clases presenciales, como en Nicaragua, la suspensión total del año escolar como en Bolivia, hasta una breve suspensión presencial con una atención aceptable de los procesos formativos aprovechando las TIC, como en Uruguay (Organización de las Naciones Unidas para la Ciencia, la 
Educación y la Cultura -Unesco, por sus siglas en inglés Unesco- Sistema de Educación de Tendencias Educativas en América Latina; 2020).

En el caso de Colombia, según la Organización de las Naciones Unidas para la Ciencia, la Educación y la Cultura (Unesco) para el 2 de agosto del 2020 se estimó que 12.842.289 de estudiantes, de los cuales 4.303.833 están ubicados en Educación Primaria, se vieron afectados por la situación descrita. Sin embargo; el país tiene la responsabilidad de garantizar los Derechos Básicos de Aprendizaje (DBA), tal como lo instituye el Ministerios de Educación, entendidos estos como:

El conjunto de aprendizajes estructurantes que construyen las niñas y los niños a través de las interacciones que establecen con el mundo y por medio de experiencias y ambientes pedagógicos en los que está presente el juego, las expresiones artísticas, la exploración del medio y la literatura (Colombia Aprende, párraf. 1)

Así, los DBA se insertan para su operacionalización en las Mallas de Aprendizaje, lo que permite integrar de manera organizada los conocimientos epistemológicamente constituidos y los Estándares Básicos de Competencias, en una secuencia de complejidad progresiva a lo largo de los años que forman cada nivel del sistema educativo. No obstante, en el escenario de la educación de emergencia ¿cómo avalar este conjunto de aprendizajes? Corresponde al docente planificar, desarrollar y evaluar el proceso formativo, lo cual lleva a plantear la urgencia de que ellos posen una mirada cercana en su propia práctica, no solo para saber qué pasa, cómo y por qué, sino más importante aún, cómo puede mejorarse en su contexto específico de acción.

Con esas ideas en mente, se hace notar que en la actualidad es indispensable sistematizar la práctica docente en la educación de emergencia, tal como está aconteciendo, por lo que se ha seleccionado como escenario educativo natural a la Institución Educativa República de Venezuela, situada en el Barrio Belén Las Violetas, en la ciudad de Medellín, de manera que se realice un esfuerzo reflexivo como el que plantean Villalobos y Pedraza (2012) por "capturar los significados de la acción y sus efectos; donde a través del uso de las experiencias, la teorización y cuestionamiento contextualizado de la praxis social, con el propósito de comunicar el conocimiento producido" (p. 347) se pueda mirar de forma colectiva y cercana la realidad de los docentes de esta institución ante esta nueva situación, documentar sus prácticas y extraer saberes. Lo que se propone es el estudio de los problemas prácticos cotidianos que viven sus maestros o profesores, en lugar de disquisiciones teóricas circunscritas a una visión positivista generadas por investigadores académicos para contribuir con el orden de una disciplina; y no es porque se descalifique el valor de este conocimiento, sino porque es relevante conocer si hay una verdadera interiorización, o las soluciones emergen de otras consideraciones asociadas con el contexto y condiciones sociales, culturales e históricas diferentes, inesperadas y apremiantes como las que han surgido en esta nueva realidad.

Es pertinente traer esta reflexión de Zemelman (2005) que, aunque fue pensada años antes de la declaración de pandemia, retrata la urgencia de forma certera: 
Estamos obligados a comprender que no es suficiente con solo explicar los fenómenos, sino que hay que transformarlos en espacios de posibilidades para reconocer opciones de otras prácticas sociales vinculadas a sujetos todavía no reconocidos. Quizás la complejidad del actual contexto resida en lo inesperado en relación con el pasado, incluso con el pasado más inmediato, ya que puede ser de discontinuidad profunda; de ahí que estemos en una situación de carencia de nombres apropiados. Y reconocer que estamos enfrentados a situaciones no solamente desconocidas sino inciertas, exige un esfuerzo sin precedentes de voluntad de conocer, propio de los momentos caracterizados por los grandes cambios revolucionarios (p. 14).

Sí en ese momento la discontinuidad aludida tuvo como punto de inflexión la creciente presencia de las Tic y su inserción en el proceso formativo, en el año 2020 y a raíz de las medidas de distanciamiento físico que obligaron a una presencia y "migración" improvisada y repentina hacia los ecosistemas y recursos tecnológicos, los agentes educativos en su mayoría quedaron simplemente perplejos. Sin embargo, es oportuno traer a colación el llamado de atención de Olivares (1987), en cuanto a la inadmisibilidad de la sumisión ante el devenir, puesto que el futuro resulta de las acciones acometidas en el presente, así que la actitud debe ser hacia la aceptación completa de la situación para conocerla y poder participar, es decir, asumir como punto de partida un contexto que se requiere "comprender en su integridad y con la convicción de que podemos contribuir a definir nuestro propio futuro...debemos investigar sobre nuestra situación y adelantar orientaciones que nos encaminen a consolidar respuestas inteligentes para nuestros problemas educativos" (p. 112).

Es en este escenario donde resulta impostergable implementar una estrategia de intersección de saberes y de prácticas entre docentes, que es posible desde la reflexión no solo entendida como una consideración detenida y aguda sobre la propia acción lo cual ya es relevante, sino que se asume como en el lenguaje de programación, como la capacidad de modificar su estructura fundamental y ejecutiva, que en el proceso de formación implica la concepción y fundamento del aprendizaje, toma de decisiones y actuación pedagógica, para que gracias a este proceso, lo aprendido por los maestros y profesores se transforme durante la transferencia, y en la reflexión de la experiencia, se revierta en un tomar consciencia y adecuar la práctica mientras nuevamente se autoevalúa, entrando así en una nueva fase de este bucle recursivo.

\section{¿Qué Es La Práctica Del Docente?}

La práctica docente es en esencia el actuar docente en relación con los estudiantes a propósito de determinados contenidos. Esto significa que existe una triada: docente, estudiante y contenido, y en esta se interactúa alrededor de narrativas y acciones, generando un conocimiento práctico que se identifica como saber, que tal como lo expresan Cañedo y Figueroa (2013) en este está inmerso la concepción del profesor sobre todos los aspectos que se activan en el proceso formativo: currículo, didáctica, aprendizaje, gestión en el aula y clima afectivo. 
Es en ese quehacer que se va demarcando una ruta que puede ser repensada, considerando el proceso y sus resultados, con base en evidencias que son importantes recabar, para simultáneamente favorecer la reflexión del docente sobre: (a) las propias acciones, la forma que las actualiza, reconsidera, ajusta y proyecta en el futuro; (b) el marco institucional en el que se desempeña y al cual está sujeto según el modelo y proyecto educativo; (c) el espacio interpersonal que está asociado al ambiente en el cual se genera la interacción entre los agentes principales del proceso, influido por sus características y propósitos en la situación de aprendizaje; y finalmente, (d) el contexto social, económico y cultural que hace patente las condiciones de ventajas y desventajas por lo cual es necesario implementar estrategias de compensación (Fierro, Fortuol y Rosas, citados en Cañedo y Figueroa, 2013)

Sin embargo; las acciones de los docentes según Cataneo (2015) pasan por:

Diseñar escenarios de aprendizaje, procesos de formación que lleven a experiencias significativas, ser un guía con una metodología centrada en el desarrollo del alumno, evaluar todo el proceso de aprendizaje, también deben autoevaluarse, es decir, reflexionar acerca de su docencia y de sus propias necesidades de aprendizaje para lograr cambios y hacer sostenible la mejora educativa... Los profesores, cuentan con una carga de trabajo grande como programar y planear todas sus clases frente a grupo, brindar asesorías y tutorías, actividades extracurriculares, entre otras, aunado a esto se suma ...cursos de actualización y de formación continua... (p. 28)

Eso resalta que el hablar de la práctica docente solo tienen sentido si se expresa como un hacer que es también social y cultural, que se da, por tanto, en un contexto específico con sus propias condiciones, dentro de una tradición concreta en la cual se participa, aconteciendo como una experiencia histórica. Es por esto por lo que, García et al (2015) afirman:

La escuela como institución, permea y encarna lo que hace el maestro, por lo que la práctica docente incluiría no solo las relaciones entre el docente y sus estudiantes, sino también las relaciones que establece con la institución, con las familias y la comunidad; los implícitos y los explícitos; sus concepciones sobre el quehacer docente y sus formas de realizarlo; sus saberes disciplinares y la transposición didáctica que realiza sobre los mismos; las formas en que enseña y las herramientas de las que se vale; los instrumentos con los que evalúa y lo que concibe como evaluación... incluso los aprendizajes ... y las experiencias que lo encarnan.

Pero el docente no es un sujeto desprovisto de decisión, todo lo contrario, en este marco él genera acciones y propuestas didácticas con miras al aprendizaje de sus estudiantes, las evalúa y reflexiona sobre ellas para transformarlas y cualificarlas... trasciende al mero hacer ...para transformarse en ... un sujeto capaz de construir saber pedagógico, de transformar su práctica y de formar críticamente a sus estudiantes (p. 13).

Según Kemmis (citado en Valladares, 2015), la práctica docente implica: un contexto, lo que hace necesario la descripción de lo micro y macro, entendiendo que se hace referencia a la relación espacio-tiempo, es decir, el dónde, desde cuándo, cómo ocurre, a quiénes afecta 
y cómo influyen los marcos institucionales; un saber hacer (Know how) por el cual se aborda lo que ocurre, quiénes lo hacen, cómo lo hacen; la valoración de significados y creencias, o sea, por qué lo hacen de esa manera, cuál es la representación del otro. Esto se realiza a través del discurso, el cómo los actores describen, interpretan y justifican esas prácticas; y, los recursos que posibilitan o limitan las acciones y el logro de los fines (inventario). Es así por lo que, considerar la práctica docente en tiempos de educación de emergencia pasa por un cambio trascendental, en especial pero no de manera exclusiva, para los maestros de educación preescolar y primaria, quienes por lo común consideraron, cuando era posible el uso de las TIC de forma más o menos complementaria y restringida, por razones neuroeducativas y de limitados recursos. Sin embargo, en el presente y posiblemente en el futuro (así sea de forma intermitente), será una de las principales maneras de poder brindar continuidad al servicio educativo.

\section{La Práctica Docente Y El Uso De Las Tic}

Según establece Coll (2011), en las investigaciones respecto al uso de las TIC en educación durante la primera década del nuevo milenio, se detectan las siguientes categorías:

1. A modo de herramientas intermediarias entre los estudiantes y los contenidos (y estrategias) para el aprendizaje.

2. A modo de herramientas de mediación entre los educadores y los contenidos (y estrategias) formativas.

3. A modo de herramientas mediadoras de la interacción entre los profesores y los estudiantes o de los alumnos entre sí.

4. A modo de herramientas de acción concertada por educadores y estudiantes durante la realización de las tareas o actividades formativas.

5. A modo de herramientas estructuradoras de ecosistemas de trabajo y de aprendizaje.

No obstante; el contexto mundial de hoy es muy diferente, y lo que parece estar sucediendo es que esa tipología se está conjugando, en todas las vertientes posibles y de manera simultánea, entre los agentes educativos que tienen acceso a herramientas y plataformas web o a aplicaciones que pueden adaptarse en su uso a los fines formativos. Sin embargo, lo que está aconteciendo entre los maestros y estudiantes en su mayoría, no sucede de una forma organizada ni en correspondencia con las pautas que aportan las investigaciones que desde hace mucho se vienen realizando acerca de la Educación a Distancia (EaD), abarcando esta, como lo señala García-Aretio (2003), desde la enseñanza por correspondencia, la educación radiofónica, el aprendizaje autodirigido por medio de módulos instruccionales, teleformación (clases por televisión abierta y por teléfonos inteligentes) hasta la formación semipresencial, la educación virtual (on line) y el e-lerning; cobrando en la actualidad nueva vida las formas más antiguas no dependientes de plataformas en la web, e incrementando las demandas de conexión y equipos de tecnología sofisticada, para restablecer y continuar brindando el servicio educativo al mayor número de estudiantes posibles, con docentes que desarrollaron sus competencias didácticas en y para ambientes presenciales. 
Es necesario entonces, conocer qué se ha aprendido y detectar los vacíos e incertidumbres que se han hecho patentes en esta jornada, para tomar decisiones y emprender acciones más certeras respecto a todas esas vertientes de la $\mathrm{EaD}$, entendiendo que todas son tecnologías aun cuando no todas son TIC, es decir, las que dependen de la web y plataformas electrónicas, y estas son de especial importancia porque es allí donde se había observado la mayor resistencia de los docentes en la incorporación en sus prácticas, pese a que su uso favorece el desarrollo de competencias del siglo XXI cuando forman parte de una estrategia didáctica integralmente concebida y desarrollada (Poblete, 2015)

Lo referido demuestra no solo las múltiples responsabilidades implícitas en la misión docente, sino que además resalta lo valioso de rescatar desde la reflexión en la acción, a los saberes pedagógicos que se ponen en marcha y devienen del hacer de los educadores en el proceso formativo, y cobra mayor importancia desde la irrupción del Covid-19 al obligar a asumir una educación de emergencia, en la cual el aula presencial ha sido sustituida por Entornos Virtuales de Aprendizaje (EVA) en distintas modalidades, unas más estructuradas que otras, pero que con toda seguridad, su estudio sistemático puede ayudar a convertir los aciertos y las equivocaciones en capacidades institucionales y comunidades profesionales, gracias al trabajo en redes de docentes reflexivos (Bolívar, 2020).

\section{La Práctica Docente En La Educación Emergente}

Con lo expuesto hasta ahora queda claro que es tiempo de ir pasando de una educación de emergencia a una emergente, es decir, aquella que incorpora de forma armónica el uso de las TIC para propiciar el proceso de aprendizaje significativo en los estudiantes. Para que esto sea posible, urge incorporar acciones precisas como las planteadas en la "lista de requerimientos para la docencia remota" de la organización Quality Matters (2020), en la cual se presentan una serie de puntos para que la triada docentes-estudiantes-contenidos (que como ya se señaló, son la base de la práctica docente), tenga mejores resultados. Estos se enumeran a continuación dentro de las categorías respectivas:

A. Introducir al estudiante

1. Brindar indicaciones precisas a los estudiantes sobre cada uno de los aspectos que componen su curso, identificarlos con precisión y en qué espacio del entorno virtual puede encontrarlo.

2. Especificar las vías y pautas de comunicación síncronas y asíncronas.

3. Aclarar los tiempos que se tardará en responder dudas, evaluar trabajos y realizar reorientaciones.

4. Indicar la manera específica de acceder a las calificaciones.

5. Informar con exactitud las fechas y horas tope de entregas y cualquier cambio que surja al respecto.

6. Ofrezca el contacto de apoyo tecnológico con el que puede contar, así como las herramientas que deberán comprar o utilizar el estudiante durante el curso y los tutoriales pertinentes. 
7. Enlazar de forma expedita y fácil instancias de apoyo para el servicio estudiantil, como las bibliotecas o con los encargados de orientar sobre la accesibilidad para atender a estudiantes con diversidad funcional.

B. Orientación del proceso de aprendizaje de los estudiantes

1. Generar actividad de presentación o de socialización con tono afectivo o de convivencia en el ecosistema formativo, con algún recurso o herramienta que se vaya a utilizar en el curso en repetidas ocasiones, por ejemplo, el foro.

2. Abrir el tema con recursos como un breve vídeo (2 a 3 minutos, si son alrededor de 10 minutos es mejor fraccionarlo) o texto realizado por el docente, en el que se conecte el discurso con el objetivo de aprendizaje.

3. Precisar la conexión entre cada actividad, la evaluación y el objetivo de aprendizaje o las competencias que moviliza.

4. Realimentar con reconocimientos o reorientar en busca de mejoras, de forma oportuna y específica, el desempeño y/o logros, usando medios variados y criterios explícitos que le sean útiles al estudiante.

C. Previendo la prolongación o intermitencia del escenario de educación de emergencia

1. Pensar en los cursos o proyectos como un conjunto de componentes completos cada uno por sí mismo, de manera que permitan su aprovechamiento en distintos contextos: presencial, híbrido, En-Línea o virtual, según la situación lo demande.

2. Diseñar con una estructura tecnopedagógica como guía, es decir, con un orden para las actividades y los recursos, con espacios suficientes para: orientar y/o socializar durante el lapso académico, y para consultar materiales multimedia y evaluar, por sesión o semana, según la secuencia didáctica.

3. Orientar sobre la privacidad, seguridad y protección de la información en los sitios web y en el EVA y su sala de videoconferencias (si la hay) en particular.

4. Utilizar textos, imágenes y recursos de elaboración propia, con licencia adquirida o de libre uso con licencias Creative Commons, y modelar en los estudiantes la virtud de la integridad académica.

Ahora bien ¿cómo se ha estado realizando la introducción al estudiante y el desarrollo del proceso formativo en estos entornos? Esta interrogante debe ser atendida en la medida que se está desarrollando esta transferencia en un contexto en principio no planificado, pero que hay que comenzar a proyectar para los próximos lapsos académicos. Es por ello que resulta oportuno iniciar esta indagación desde una sistematización de experiencias en primer término, para luego plantear una investigación-acción-participante, es decir, una combinación de dos métodos que son compatibles porque ambos, aunque se encuentran cercanos a la tradición cualitativa, no pretenden interpretar la realidad sino abordarla de forma crítica, primero para conocerla y poder así discernir saberes; y, luego para transformarla de manera que se puedan garantizar los DBA.

Tal como hallaron García-Cepero y cols. (2015) en su estudio sobre las rutas de emergencia en el trabajo docente a través del estudio de casos de maestros con uso 
sobresaliente de las TIC, los docentes deben ser capaces de reflexionar críticamente sobre su práctica y sistematizar los saberes producto de estas prácticas educativa. En esto, hay que coincidir con Carr y Kemmis (1986) cuando afirman que el conocimiento está atado a las inquietudes de la vida diaria, porque devienen de las necesidades comunes de los seres humanos, que a su vez están determinados en cierta forma por el contexto sociohistórico, aunque el ecosistema tecnológico no sea natural, lo cierto es que se está generando un hacer que es importante investigar mientras se genera y transformar en tanto se investiga.

\section{Convertir La Práctica Docente En Saberes: Sistematizar Y Transformar}

El aprender de la experiencia no es una propuesta nueva. Sobre este asunto, es común entre los autores que lo proponen la cita de este extracto de la Ética a Nicómaco, de Aristóteles, en el 350 a.C. "para las cosas que tenemos que aprender antes de que podamos hacerlas, aprendemos haciéndolas", y también se le atribuye la frase "la calidad no es un acto, es un hábito", que al considerarlas juntas se deduce que, para este filósofo el aprendizaje experiencial no tiene límites. Sin embargo, no se trata de un ejercicio mecánico, sino de la búsqueda constante de un desempeño integro.

Mucho tiempo después, fue el trabajo de John Dewey, luego Kurt Lewin y posteriormente Jean Piaget quienes, cada uno en su propio contexto enfatizó la necesidad de reflexionar sobre la propia acción para aprender y convertirla en sabiduría práctica (phronesis), es decir, en ejercitación de la virtuosidad (Molina, Pardo, y Rodríguez, 2017) y una práctica social que en su momento, David Kolb contribuyó en su consolidación como una teoría del aprendizaje experiencial (León, 2015) que tiene como base la premisa de que el conocimiento se genera de la metamorfosis inducida por la experiencia, pero que no conduce a un estado fijo, sino que a la vez sufre una transformación al reconsiderar los cambios en la teoría o los postulados que animaron la actuación original, y estos se proyectan en nuevas formas en el hacer, en un proceso iterativo o por ciclos en espiral.

Según Wenger (2001) el aprendizaje experiencial abarca lo manifiesto, por lo que tiene que ver con símbolos, herramientas, papeles que se desempeñan, pautas que se determinan según la finalidad de la variedad de prácticas. Al mismo tiempo, incluye lo implícito, es decir, lo no escrito, los acuerdos tácitos, supuestos no declarados pero presentes que dan indicios y "son señales inequívocas de la afiliación a una comunidad de práctica y son fundamentales para el éxito de su empresa" (Wenger, 2001, p. 71). Lo que resulta relevante para el docente, es ir más allá con la sistematización de la experiencia de manera que se documente ese bucle iterativo, haciendo explícito lo que está afectando el proyecto educativo: lo que está funcionando y lo que se puede mejorar, para generar un aprendizaje colectivo, un acervo para la institución y para otras organizaciones educativas de contextos similares.

Esto implica un abordaje pospositivista, en el que el conocimiento científico también se genera de la reflexión sobre la práctica y su sistematización, de forma particular, cuando se habla del conocimiento pedagógico, se conjugan ciencias fácticas y formales según se refiera a sí mismo o a su transferencia en el proceso formativo de las distintas disciplinas, 
para reflejarse en una praxis que tiene como fin mejorar o transformar la sociedad, según el proyecto político subyacente. Esta relación puede observarse en la siguiente figura:

\section{Figura 1}

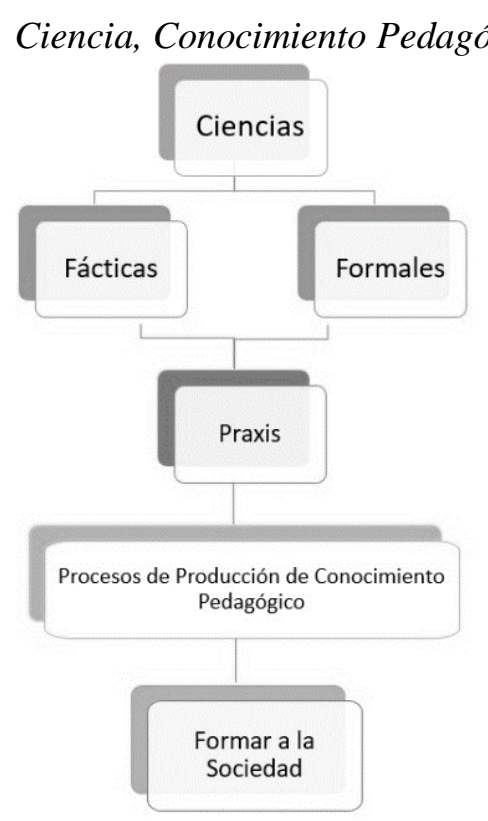

Nota: El gráfico representa la dependencia del conocimiento pedagógico respecto a las ciencias fácticas y formales y su propósito en la sociedad. Tomado de Modelos Pedagógicos (p. 45) por Román (2019), Universidad Metropolitana de Educación, Ciencia y Tecnología.

Las ciencias fácticas (Arrieta, s. f.) son las que están basadas en hechos, es decir, son empíricas y se nutren de información lograda por medio de los instrumentos que provee el conocimiento científico, con rigurosa aplicación de la lógica para que se traduzca en representaciones mentales que están en consonancia con lo representado. Estas se clasifican en naturales (las que estudian fenómenos que se dan en la naturaleza): biología, química, física, otras; $y$, sociales o culturales (indaga respecto al ser humano, sociedad e instituciones): economía, historia, sociología, antropología... y cada una de las ciencias de la educación, entre las que se encuentra la pedagogía, como su disciplina central. Por otro lado, las ciencias formales o eidéticas pertenecen al espacio epistémico de las ciencias que deducen principios y leyes por ensayos o abstracciones mentales (como matemáticas y la misma lógica).

Con respecto a la praxis, se hace alusión al "acto o conjunto de actos en virtud de los cuales el sujeto activo (agente) modifica una materia prima dada" (Sánchez, citado en Palazón, 2006, p. 241), en conjunción con una teoría que le sirve de guía por su sentido anticipatorio, a la que trasciende en la práctica (el saber cómo). No siempre existe una relación ordenada entre ambas, pero esto no impide su mutua influencia en dos direcciones: en una, la práctica da lugar a la generación de teorías por medio de inferencias; y en la otra, las proposiciones teóricas al operacionalizarse confirman, profundizan o invalidan formas de transferencias. Es así por lo que la praxis resulta ser un binomio teórico-práctico, y en lo 
personal o colectivo deviene en experiencias, que en este caso son apreciadas porque el confrontarse y ahondar equivalen a un proceso de destilación o de tamizaje en el que se concentran los saberes que representan la virtud de la sabiduría.

Para que eso sea posible en la pedagogía, es crucial la sistematización de experiencias orientadas a reconocer prácticas que pudieran o no coincidir con lo establecido, porque lo que se impone en el aula es la apropiación o la creatividad, pero también las rutinas y las exigencias que surgen desde diferentes ángulos con distintos pesos en la toma de decisiones y en el accionar, entre estas se pueden mencionar: el modelo educativo, los estudiantes, otros docentes, directivos, padres, supervisores y los eventos inesperados como la aparición del Covid19, por ejemplo, que se imponen a lo planificado. Elliot (2000) apunta que esta es una forma de abordar los desafíos diarios que surgen en el hacer y con el escenario que se presenta, en lugar de proposiciones teóricas pensadas por académicos que, no en pocas ocasiones se acercan a levantar información sobre la base de opiniones, cruzadas o no, entre diferentes actores, pero que no se confrontan con la práctica y su reflexión paulatina mientras está sucediendo el hecho educativo, y por lo tanto, desde la indagación disciplinar solo se usan para corroborar lo que se piensa que es y no lo que existe en la realidad y a qué se debe.

\section{Sistematización De La Práctica Docente}

La sistematización de experiencias en la práctica educativa como método de investigación puede definirse como la búsqueda organizada, crítica, dialógica, constante y rigurosa de respuestas sobre el qué y cómo acontece el actuar de uno o varios de los agentes formativos (gerentes, docentes, estudiantes, equipos de apoyo, comunidad educativa) con el fin de conocer y entender las dimensiones que subyacen en esta actuación, para hacerlas patentes contrastando postulados y acciones, concepciones y la lógica de la que emergen con el conocimiento teórico disponible. Como lo expresan Castaño et al. (2019) “en las dinámicas metodológicas de sistematización, son fundamentales tanto el proceso como el producto, pues ambos generan aprendizaje, saber, conocimiento y experiencia en quien o quienes la implementan." (p. 7) y, se requiere además de su divulgación y comparación posterior con otras experiencias para que sus hallazgos sean, como corresponde en el hacer ciencia, una contribución al acervo de la práctica pedagógica como área de estudio.

Para Pineda y Velásquez (2015) la sistematización de experiencias, sin importar los autores que la definan, coinciden en que requiere de "la organización, la interpretación, la reflexión, la exploración y elaboración teórica en torno a lo que se sistematiza; es decir, una sucesión de trabajos que buscan fortalecer el sentido y significado de la práctica realizada" (p. 16), que se dirige por y para los propios actores por lo que resulta en un proceso autoformativo.

Para Torres (1998) y Martínez-Gómez (2019) la sistematización se caracteriza considerando varios aspectos, que pueden relacionarse de la siguiente forma:

1. Una generación deliberada de conocimientos desde la experiencia de los sujetos (autoobservación) que problematizan su realidad en colaboración mutua, para reflexionar y 
al mismo tiempo construir explicaciones desde la crítica, en las que se parte de reconocer lo intrincado que es el fenómeno educativo y sus prácticas, por lo que se propone comprenderlas en toda su complejidad, cuestionando la rigurosidad de las premisas que sustentan el accionar de sus actores, con el fin de fortalecer su práctica formativa en tanto que contribuye con su teorización. No pretende objetividad, ya que la reflexión crítica y rigurosa toma su lugar.

2. Una concepción de la realidad, de la ciencia, la política y la educación que no pretende ser negada, sino que se asume como parte del sistema de pensamiento de cada uno de los actores, y, por lo tanto, no es neutro, intervienen en una dinámica dialéctica, por lo que estarán presentes y contribuyen en el cocrear una síntesis enriquecedora como su "resultado" siempre provisional y atado a su contexto.

3. Un método con procedimientos emergentes, como es propio de las investigaciones cualitativas, lo que resulta en una mixtura de técnicas, registros y análisis que buscan capturar no solo de forma intersubjetiva la realidad, sino que generan la información suficiente para incidir con una mirada crítica, lo que lo diferencia de otros métodos interpretativos y lo ubica en las tradiciones crítico-emancipatorias. La sistematización entonces sirve develar las relaciones y sus posibilidades.

4. El papel de quienes investigan y su relación con los sujetos. Quien investiga se involucra activamente, en un movimiento de formación y transformación conjunta con el grupo social que investiga.

5. La sistematización como reconstrucción de sentidos de la experiencia. Las prácticas sociales y educativas para sistematizar se conciben como realidades complejas, como construcciones históricas y de sentido donde confluyen factores y dinámicas objetivadas con procesos e interacciones subjetivas, que además están atravesadas por relaciones de poder. La sistematización busca dar cuenta de esa interacción entre contextos, prácticas y sentidos.

Para el desarrollo de este método de investigación, se plantean diversas fases, que en ningún momento siguen un trayecto lineal, ya que se trata de identificar en un principio desde qué perspectiva se estudiará la práctica (desde la docencia, la gestión, el aprendizaje...), que propósito o preguntas se aspira abordar, quiénes formarán parte del proceso y qué papel tendrán, cuáles serán las fuentes de información, qué instrumentos y registros servirán para sustentar las comprensiones que surgirán; luego, es importante establecer un sistema de organización e ir recuperando la información, categorizándola, analizando, volviendo nuevamente a las fuentes o sobre las preguntas para ajustar a lo que está revelando el procesamiento de los datos o los actores... Es decir, que se requiere una búsqueda de equilibrio constante entre organización e incertidumbre, porque el investigador debe dejar que el estudio sea realmente emergente.

En la Figura 2 puede observarse la propuesta de Saenz et al. (2019) para la sistematización de prácticas educativas: 


\section{Figura 2}

Propuesta analítica de sistematización de prácticas educativas

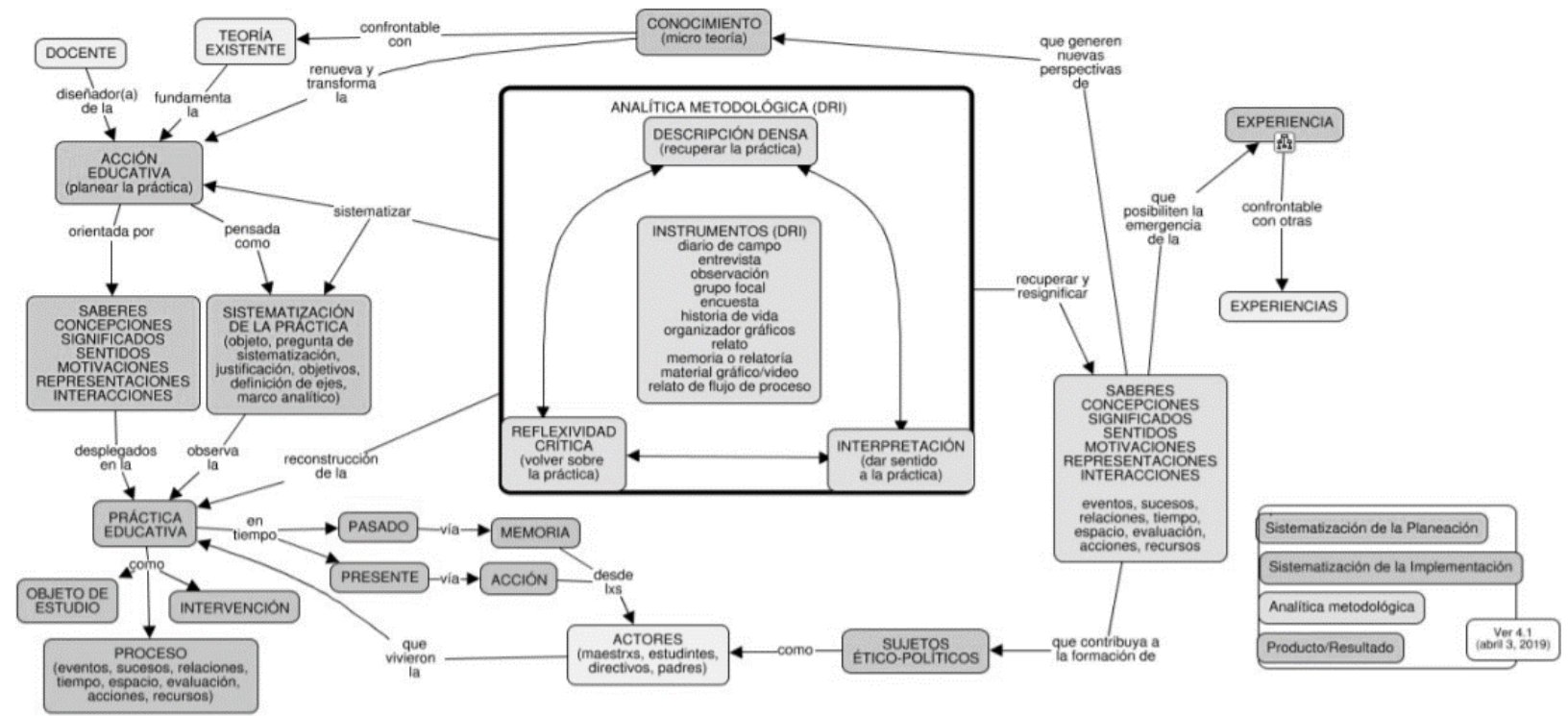

Nota: El gráfico expresa la interacción que se da entre la práctica, el conocimiento, la experiencia y los saberes en un proceso de sistematización como método de investigación. Tomado de Sistematización de Prácticas Educativas: Guía conceptual para educadores (p. 6) por Sáenz et al. (2019) Edukafé, Documentos de trabajo de la Escuela, No. 7. Cali: Universidad Icesi.

La figura anterior permite dar cuenta de lo emergente que es el método de sistematización de experiencias, que en ese equipo de investigadores en particular se sintetizó a través de ese modelo, pero que bien puede diseñarse de muchas otras formas, dependiendo de cuáles son las grandes categorías que dan dirección al estudio y la interpretación de los actores, porque es un método complejo en sí mismo que se desarrolla cuando se quiere rescatar de las prácticas los saberes, para poder hacerlos visibles y divulgarlos, en primer lugar, a los propios actores; en segundo lugar, al resto de integrantes de la comunidad educativa; y, finalmente, a otras comunidades de docentes e investigadores para hacer una validación abierta de todo el proceso y sus hallazgos.

Sin embargo; una evolución con sentido podría ser la de aprovechar los resultados de la sistematización para proponerse una intervención de la situación para generar una transformación por medio de la Investigación-Acción Participante, no porque no tengan relevancia los saberes que la práctica docente propicia, sino porque siempre es factible 
mejorar lo que se hace, y el acompañamiento del proceso de investigación brinda una mirada acuciosa que trasciende a la del docente y la de todos los participantes.

\section{Tranformar La Práctica Docente Con La Investigación-Acción Participante}

En primer lugar, es necesario precisar que la Investigación-Acción (IA) tiene un propósito básico: transformar la realidad. Por ello, es muy adecuado partir de los hallazgos de una sistematización de experiencias previa, ya que esta coloca a disposición de los agentes educativos un conocimiento certero de la situación inicial, gracias al análisis profundo, documentado, categorizado, codificado e interpretado con base en las distintas fuentes y evidencias.

Se constituye la sistematización en un punto de partida que, si bien es provisional porque requiere ser consensuado el nuevo rumbo para esta nueva etapa, tiene bases con arraigo en el propio contexto y con los mismos actores, desde donde se han podido discernir saberes, así como también áreas de mejora, que son el punto de interés para una intervención desde la IA.

La IA es definida por Lewin, quien creó esta denominación en 1946, como una vía para la autorreflexión realizada por participantes que cuestionan sus prácticas socioeducativas, establecen prioridades de intervención desde nodos críticos, y emprenden acciones que permitan conocer la realidad y sus cambios desde la racionalidad y justicia en un contexto delimitado (Latorre, 2007). Esta modalidad de investigación tiene la característica de ser hecha con la gente, por la gente y para la gente de un espacio específico, utilizando las técnicas e instrumentos que se consideren oportunos, sin importar si se tienden a usar en enfoques cualitativos o cuantitativos, ya que, su fidelidad es con el compromiso de cambio, no con un enfoque metodológico.

El tipo de conocimiento que interesa en la IA educativa es todo aquel que tiene su origen en la transferencia del conocimiento generado en la ciencia y la tecnología, pero también en la vida social y familiar, dentro del contexto aúlico, para lo que es imprescindible desarrollar una variedad de prácticas entre los actores del proceso formativo para su apropiación. Tal como lo expresa De Zubiría (2010): 
Hay que reconocer que el conocimiento se construye por fuera de la escuela, pero que es reconstruido de manera activa e interestructurada a partir del diálogo pedagógico entre el estudiante, el saber y el docente y que para que ello se presente, es condición indispensable contar con la mediación adecuada de un maestro, que favorezca de manera intencionada y trascendente el desarrollo integral del estudiante. (p. 2).

Hablar de mediación es lo mismo que referirse a prácticas, en el sentido de abordar lo que hace el docente en el aula para propiciar el aprendizaje, y es en este espacio donde la IA se dirige a la transformación como una alternativa ante las limitaciones del enfoque metodológico cuantitativo para propiciar cambios profundos. Descansa en tres ideas fundamentales: (a) el contexto ejerce influencia sobre las personas, por esto, la IA se realiza en el escenario natural en donde interactúan; (b) los espacios naturales son ricos en la información sobre la interacción de las personas porque es el lugar en donde se desarrollan las prácticas; y, (c) las metodologías cualitativas son las más apropiadas para recabar información en entornos naturales, lo cual no significa que para la IA las técnicas e instrumentos sean excluyentes entre enfoques metodológicos, tal como ya se mencionó (Cossio, 2011)

El centro de la IA es la reflexión, con base en un problema de la práctica pedagógica, es decir, aquello que muestra disonancia entre lo que ocurre y lo que debería acontecer. Se asume a través de varios ciclos, que a continuación se representan en el Modelo de John Elliot (2000), que es a su vez una adaptación a la propuesta de Lewin de 1944:

\section{Figura 3}

Modelo de Investigación Acción de John Elliot 


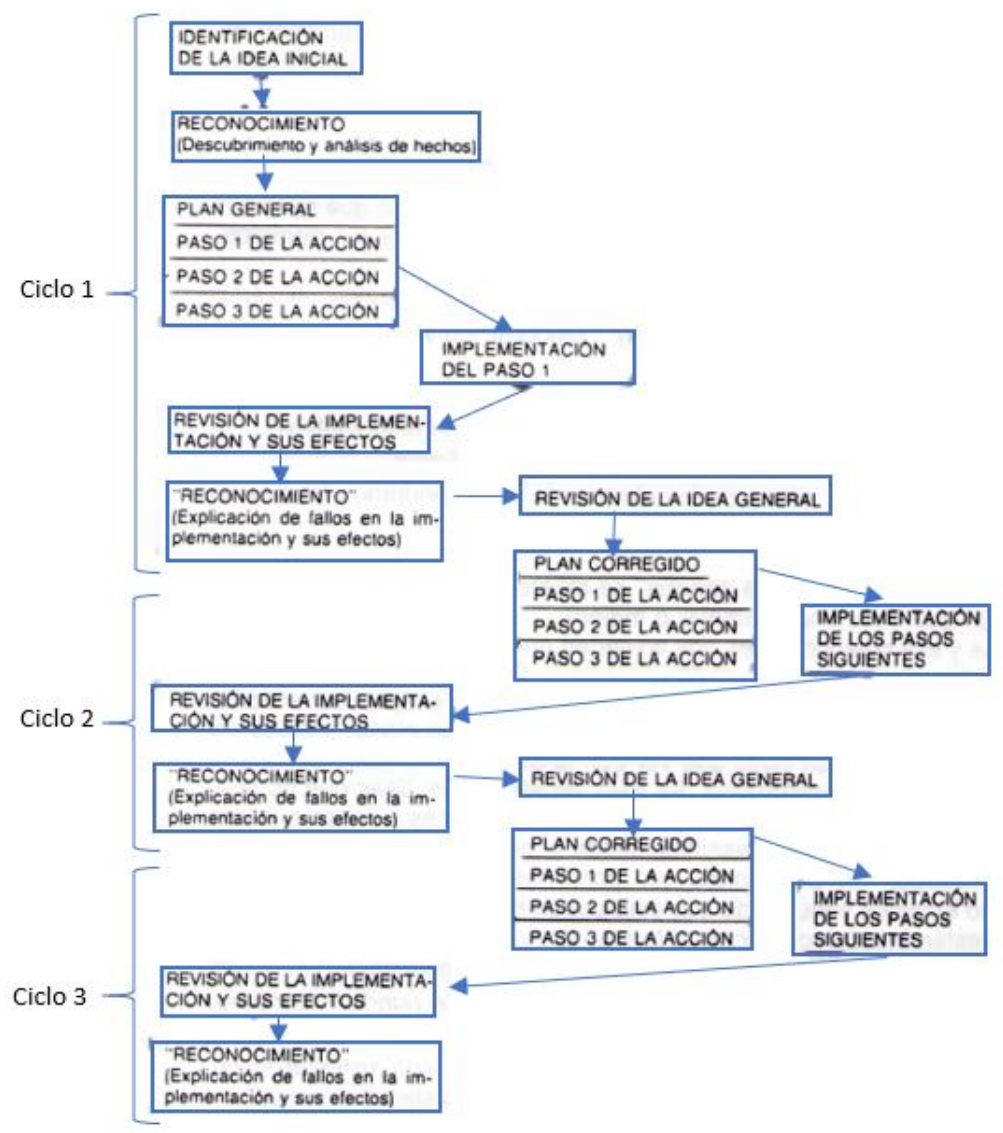

Nota: El gráfico representa los ciclos de la Investigación Acción, desde la identificación de la idea inicial hasta la culminación de la última revisión y reconocimiento de los fallos en la implementación del plan general y sus efectos. Tomado de El Cambio Educativo desde la Investigación-Acción (p. 90), por John Elliot (2000), Ediciones Morata.

Como puede observarse en el Modelo, el Primer Ciclo parte de la detección o identificación de la idea inicial, que en este documento toma como referencia la sistematización de experiencias propuesta en el punto anterior. El docente-investigador presenta una idea inicial del problema, sus causas y efectos (reconocimiento y análisis de los hechos). Un punto muy importante de aclarar es que los objetivos de la IA no son los mismos que los del plan de acción, ya que, por tratarse de un proceso científico, los objetivos generales y específicos se orientan a aportar conocimiento sobre la práctica, mientras que los del plan se dirigen a resolver la problemática. Esta distinción es fundamental. De allí se avanza a la elaboración del plan de acción y establecer los pasos de la acción, que realizan todos los involucrados utilizando diversas matrices e instrumentos de análisis, como: matriz de priorización, diagrama de Ishikawa, Árbol de Problema, entre otros, lo que permite 
determinar las prioridades, para lo cual se consideran: importancia, viabilidad y potencial de mejoramiento, en todo momento.

Llega el momento de la ejecución de los pasos de la acción 1; y se inicia la primera acción que se ha establecido, acompañada de una mirada cercana que implica un monitoreo de cada uno de los pasos, durante la cual se recaba información a través de los instrumentos que sean pertinentes, para verificar los aciertos y desaciertos esta primera acción en cuanto a logros y desempeños, lo cual lleva al reconocimiento de los fallos y sus efectos, para una revisión de la idea general.

El Segundo Ciclo parte de los ajustes a al plan general, se prosigue con los pasos de la acción No. 2, la documentación y reflexión acerca de los logros y errores en esta nueva acción con los instrumentos y sobre la base de las evidencias, en una nueva revisión y corrección del plan general, para continuar con el Tercer Ciclo del modelo, implementando los pasos de la acción No. 3, la recolección de datos sobre lo que está aconteciendo como consecuencia de la intervención, una nueva valoración del proceso y los productos, para un nuevo reconocimiento de la afectación del contexto. Este proceso se repite hasta que se documenta la transformación, aunque también busca que el docente interiorice a la investigación sobre su propia práctica de forma permanente, y es característico de todo el proceso. Entonces, se puede deducir que los objetivos son más estables, el plan de acción, no (Elliot, 2000).

Lo esencial al asumir la IA como acercamiento metodológico a la realidad, es la participación auténtica de todos los agentes educativos a quienes concierne y afecta el proceso, ya que es parte la axiología que involucra. Por ello, es necesario tener presente a que "la participación auténtica si incluye representantes de grupos de interés relevante y crea espacios relativamente seguros y estructurados para que las diferentes voces sean escuchadas y se orientan a los fines de la participación” (Anderson, G. 2005, p. 8).

\section{Conclusiones}

La educación que se brinda a partir de la pandemia por Covid-19 es una réplica de la situación de emergencia global: llena de incertidumbres en la que muchos eventos 
simultáneos hacen difícil distinguir causas de efectos, por tanto, es caótico y sin tiempo para las respuestas exactas; esto lleva a afirmar que solo puede ser asumida desde el pensamiento complejo en el cual las soluciones nacen de las mismas circunstancias, son los actores con sus decisiones en atención a lo que aparece, y esto, en particular, ha recaído sobre los hombros de los docentes, quienes han tenido que interpretar y adaptar los lineamientos transferidos a sus prácticas, que en el caso que sirve para ilustrar este escrito se hace referencia al personal académico de la Institución Educativa República de Venezuela.

Pero ¿cómo saber cuáles han sido los aciertos y los errores en la práctica? Para responder a esta interrogante se ha propuesto a la sistematización de experiencias en una primera aproximación a esta realidad, para luego realizar una Investigación-Acción orientada a transformar la práctica de los docentes con el fin de brindar una educación que contribuya a cerrar, o al menos, a no profundizar, la brecha social.

\section{Referencias}

Adell, J. y Castañeda, L. (2013) El ecosistema pedagógico en los PLEs. en red. Alcoy: Marfil

Anderson, G. (2001) Hacia una participación auténtica: Deconstruyendo los discursos de las reformas participativas en educación. En Narodowski, N.; Nores, M. y Andrada, M. (eds.) Nuevas tendencias en políticas educativas. Temas/Fundación Gobierno \& Sociedad.

Arrieta, E. (s. f.) Ciencias naturales y ciencias sociales. El diferenciador. En https://www.diferenciador.com/ciencias-naturales-y-ciencias-sociales/

Bain, K. (2007) Lo que hacen los mejores profesores de la Universidad. Traducción de Óscar Barberá. Universitat de Valéncia

Bandura, A. y Walters, R. (1974). Aprendizaje social y desarrollo de la personalidad. Alianza Universidad

Blandler, R. y Grinder, J. (1997) La estructura de la magia I. Editorial Cuatro vientos.

Bolívar, A. (2020) Capacidades institucionales para la autoevaluación. Sistema Nacional de Evaluación, Acreditación y Certificación de la Calidad Educativa, Sineace.

Cañedo, T. y Figueroa, A. (2013). La práctica docente en educación superior: una mirada hacia su complejidad. Sinéctica, (41), 2-18. http://www.scielo.org.mx/scielo.php?script=sci_arttext\&pid=S1665109X2013000200004\&lng=es\&tlng=es.

Castaño, A.; Sáenz, D; Ávila, C.; Bianchá, H.; Segura, J.; y, López-García, J. (2019) Sistematización de Prácticas Educativas: Guía conceptual para educadores. Edukafé, 
Documentos de trabajo de la Escuela, No. 7. Cali: Universidad Icesi. Recuperado de Eduteka: http://doi.org/10.18046/edukafe.2019.7

Cataneo, L. (2015). Las dimensiones de la nueva práctica docente en la era del conocimiento y la información: El desarrollo profesional del profesor y su transferencia del conocimiento. Tecnológico de Monterrey. En: https://repositorio.tec.mx/bitstream/handle/11285/622360/02Luis+Rodolfo+Cataneo+Flore s.pdf? sequence $=1$

Coll, C. (2009). Aprender y enseñar con las TIC: expectativas, realidad y potencialidades. Artículo incluido en: R. Carneiro, J. C. Toscano y T. Díaz (compiladores). Los desafíos de las TIC para el cambio educativo. OEI-Santillana, Fundación Santillana, pp. 113-126.

Cossio, C. (2011). Investigación Acción Educativa. Videoconferencia de la Formación Inicial Docente, en: https://youtu.be/fYuQ04_W3wc

De Zubiría, J. (2010). A refundar la escuela. Asociación de Educadores Latinoamericanos y el Caribe.

Díaz y Pinto: Vulnerabilidad educativa: Un estudio desde el paradigma socio crítico. Praxis educativa, Vol. 21, No 1; enero-abril 2017- ISSN 0328-9702 (impreso) y 2313-934 X (en línea), pp. 46-54 DOI: http://dx.doi.org/10.19137/praxiseducativa-2017-210105

Díez-Gutiérrez, E. (2020). Otra investigación educativa posible: investigación-acción participativa dialógica e inclusiva. Márgenes, Revista de Educación de la Universidad de Málaga, 1 (1), 115-128 DOI: http://dx.doi.org/10.24310/mgnmar.v1i1.7154

Elliot, J. (2000). El cambio educativo desde la Investigación-Acción. Ediciones Morata.

García-Aretio, L. (2003) La educación a distancia: una visión global. Boletín Ilustre Colegio Oficial de Doctores y Licenciados en Filosofía y Letras y en Ciencias Madrid. 146.

García-Cepero, Gómez-Hernández, Barrios-Martínez, Santamaría, Sánchez, Castro y Zuluaga (2015). Rutas de emergencia en el trabajo docente. Estudio de casos en maestros con uso sobresaliente de las TIC. Universidad Javeriana, Facultad de Educación y Fundación Telefónica y Movistar.

Latorre, A. (2007). La Investigación-Acción. Conocer y cambiar la práctica educativa. Editorial Graó.

León, L. (2015). El aprendizaje experiencial de la estadística en base a los estilos de aprendizaje del estudiante universitario. UCV-Scientia, 2(2), 111-117. En: https://dialnet.unirioja.es/descarga/articulo/6181497.pdf

Martínez-Gómez, N. (2019). Retos y aprendizajes de la sistematización de experiencias de educación popular. El caso de la Pre-Universidad Tunjuelo Popular en Bogotá. Revista Brasileira de Educação, 24, e240064. Epub December 05, 2019.https://doi.org/10.1590/s1413-24782019240064 
Ministerio de Educación de Colombia, Colombia Aprende (s. f.). Derechos Básicos de Aprendizaje. En http://aprende.colombiaaprende.edu.co/es/node/94184

Molina, R., Pardo, L. y Rodríguez, L. (2017). Las maletas viajeras: significado y construcción del aprendizaje experiencial. En: https://revistas.usb.edu.co/index.php/cienciashumanas/article/view/2970

Olivares, M. (1987). Algunas reflexiones sobre el proceso de formación docente en América Latina. Innovación Educativa y Formación de Docentes, 10-120.

Orozco, I. y Moriña, A. Prácticas Docentes para una Pedagogía Inclusiva en Educación Primaria: Escuchando las voces del Profesorado. Aula Abierta. Volumen 48, número 3, julioseptiembre, 2019/págs. 331-338

Palazón, M. (2006). La praxis en la filosofía de Adolfo Sánchez Vázquez. Andamios, 3(5), 237-256. Recuperado en 18 de agosto de 2020, de http://www.scielo.org.mx/scielo.php?script=sci_arttext\&pid=S187000632006000200012\&lng=es\&tlng=es.

Pineda y Velásquez (2015) La sistematización de experiencias educativas desde el enfoque praxeológico: experiencia educativa: América Latina ¿libre, innovadora y productiva? Una revisión mediada por el ejercicio de análisis coyuntural. Corporación Universitaria Minuto de Dios. Vicerrectoría Regional Llanos. ISBN 978-958-763-135-7

Poblete, R. (2015) Habilidades del pensamiento en el siglo XXI con el uso de TIC para el aprendizaje de matemática. Repositorio Uchile. Universidad de Chile

Pontífica Universidad Católica de Chile (2018). Las neuronas espejo y su posible conexión con la empatía. Escuela de Medicina, Facultad de Medicina. En:

https://medicina.uc.cl/noticias/neuronas-espejo-y-empatia/

Quality Matters (2020) Docencia remota de emergencia. En: https://www.fernandosenior.com/docencia-remota-de-emergencia/

Torres, A. Reflexiones sobre la práctica reciente. Ponencia presentada al Tercer Congreso Iberoamericano y Caribeño de Agentes de Desarrollo Sociocultural y Comunitario. Universidad Pedagógica Nacional. La Habana, octubre de 1998.

Unesco (2 de agosto de 2020) Impacto del Covid-19 en la educación. En https://es.unesco.org/covid19/educationresponse

Unesco, Sistema de Educación de Tendencias Educativas en América Latina (21 de septiembre de 2020) Sistematización de respuestas de los sistemas educativos de América $\begin{array}{lllll}\text { Latina a } & \text { la } & \text { crisis }\end{array}$ https://www.siteal.iiep.unesco.org/respuestas_educativas_covid_19

Valladares, L. (2017). La "práctica educativa" y su relevancia como unidad de análisis ontológico, epistemológico y sociohistórico en el campo de la educación y la Pedagogía. 
Perfiles Educativos, XXXIX (158), 186-203. ISSN: 0185-2698. En: https://www.redalyc.org/articulo.oa?id=132/13253901011

Van de Velde, H. (2008). Sistematización. Centro de Investigación, Capacitación y Acción Pedagógica. https://issuu.com/ingridcitavera/docs/sistematizacion_de_experiencias_herman___1_

Villalobos, G. y Pedroza, R. Sistematización de la práctica socioeducativa. I Congreso Virtual Internacional sobre Innovación Pedagógica y Praxis Educativa: INNOVAGOGÍA 2012. Memorias. ISBN 978-84-616-1780-7

Zemelman, H. (2005). Voluntad de conocer: el sujeto y su pensamiento en el paradigma crítico (Vol. 47). Anthropos Editorial. 